\title{
Miocardiopatía por amiloidosis
}

\author{
Cardiomyopathy due to amyloidosis
}

\author{
Daniela Cabrera Palos, ${ }^{*}$ Enrique Juan Díaz Greene, ${ }^{\ddagger}$ Federico Leopoldo Rodríguez Weber ${ }^{\S}$ \\ Citar como: Cabrera PD, Díaz GEJ, Rodríguez WFL. Miocardiopatía por amiloidosis. Acta Med \\ Grupo Angeles. 2021; 19 (2): 244-252. https://dx.doi.org/10.35366/100449
}

\section{Resumen}

La amiloidosis cardiaca es una enfermedad de escasa sospecha clínica por su baja incidencia, sin embargo, en los últimos años y gracias a los adelantos en métodos de imagen, ha sido notable el aumento de casos diagnosticados, lo cual se ve reflejado en los avances en el tratamiento de los diferentes tipos de este trastorno.

Palabras clave: Amiloidosis cardiaca, cardiopatía, cadenas ligeras, transtiretina.

\section{INTRODUCCIÓN}

La amiloidosis sistémica es un conjunto de enfermedades causadas por depósito de proteínas fibrilares mal plegadas, cambiando su estructura terciaria a una más lineal, ${ }^{1}$ estos cúmulos se encuentran en el espacio extracelular de varios tejidos y órganos, lo cual lleva al mal funcionamiento y destrucción de los mismos, ${ }^{2}$ tal proceso podría ser influenciado por numerosos factores, como la presencia de proteínas amiloides inestables, aumento crónico en las concentraciones de proteína o edad avanzada. ${ }^{3}$ El término amiloide fue descrito por primera vez en 1854 por Rudolf Virchow, al observar bajo el microscopio la semejanza de la sustancia con el almidón. ${ }^{4}$ El involucro cardiaco es el factor pronóstico más importante en pacientes con este trastorno, en el cual puede haber infiltrados por inmunoglobulina de cadena ligera y por transtiretina, principalmente, la implicación de este órgano es la mayor causa de morbimortalidad en amiloidosis sistémica. ${ }^{5}$ El dictamen de

\footnotetext{
* Médico residente de medicina interna del Hospital Ángeles Pedregal.

₹ Profesor titular del curso de residencia de medicina interna del Hospital Ángeles Pedregal.

$\S$ Profesor adjunto del curso de residencia de medicina interna.
}

Facultad Mexicana de Medicina de la Universidad La Salle, Ciudad de México.

\begin{abstract}
Cardiac amyloidosis is a disease of scarce clinical suspicion due to its low incidence, however, in recent years and thanks to advances in imaging methods, there has been an increase in diagnosed cases, which is reflected in new treatments for different types of this illness.
\end{abstract}

Keywords: Cardiac amyloidosis, cardiomyopathy, light chains, transthyretin.

este trastorno cardiaco podría ser complicado, ya que el cuadro clínico no suele ser específico, se necesita una alta sospecha clínica y la falta de familiaridad puede retrasar el diagnóstico hasta estadios finales. ${ }^{6}$

\section{PATOFISIOLOGÍA}

De acuerdo con la extensión de la enfermedad, puede ser clasificada de la siguiente manera: sistémica o localizada, adquirida o heredada, o por proteínas amiloides. ${ }^{2}$ La nomenclatura utilizada para los diversos tipos de amiloidosis es $A X$, donde $A$ significa amiloidosis y $X$ representa la proteína encontrada en la fibrilla. ${ }^{4}$ La variante cardiaca se caracteriza por infiltración extracelular a través del corazón, lo cual resulta en hipertrofia biventricular con remodelamiento concéntrico, lo cual causa bajo gasto cardiaco. Dentro de las alteraciones que causa esta enfermedad se encuentran: aumento en la presión auricular asociada con dilatación de la misma, reducción de la perfusión miocár-

Correspondencia:

Daniela Cabrera Palos

Correo electrónico: danicabrerap@gmail.com

Aceptado: 10-06-2020.

www.medigraphic.com/actamedica 
dica por infiltrado amiloide, arritmias atriales o retraso en la conducción auriculoventricular, infrecuentemente se presentan arritmias ventriculares.

\section{CLASIFICACIÓN}

Los principales subtipos de amiloidosis sistémica se clasifican basados en la etiología de base: primaria, secundaria o reactiva, por transtiretina (hereditaria) o tipo silvestre (antes denominada senil) ${ }^{8}$ y relacionada a diálisis; de éstas, la primaria, la causada por transtiretina, en su forma hereditaria y silvestre, son las que afectan al miocardio, mientras que la secundaria raramente lo hace (Tabla 1). ${ }^{9}$

\section{AMILOIDOSIS PRIMARIA O DE CADENAS LIGERAS}

La amiloidosis de cadenas ligeras es una condición adquirida sin componente genético, ${ }^{5}$ la cual se produce secundaria a un desorden proliferativo de las células plasmáticas o de células B clonales, en las que tales cadenas son depositadas a guisa de fibrillas amiloides en múltiples órganos; la producción de cadenas ligeras lambda es tres veces mayor a la producción de las cadenas ligeras kappa. ${ }^{6}$
La mayoría de los casos se asocian con discrasias sanguíneas, específicamente desórdenes proliferativos de células plasmáticas. En mieloma múltiple, de $10 \%$ a $15 \%$ de los casos progresan a cadenas ligeras, mientras que otras condiciones hematológicas como macroglobulinemia de Waldenstrom y linfoma no Hodgkin, aunque se asocian menos con amiloidosis, han demostrado formación y depósito del mismo. ${ }^{3}$

Este tipo de amiloidosis es el más frecuente, presentándose en un $70 \%$ de los $\operatorname{casos}^{2}$ y es la que más afecta al sistema cardiaco. En México, entre los años 1983 a 2012, se llevó a cabo la recopilación de casos de la variante de cadenas ligeras, primarias y generalizada en el Centro de Hematología y Medicina Interna de Puebla, en donde se encontró que la incidencia es 14 veces menos frecuente comparado con poblaciones caucásicas y representa el 15\% de todas las gammapatías monoclonales. ${ }^{10}$ La alteración en la función del corazón es resultado de la infiltración extracelular, empero, hay evidencia de que también ocurre un efecto cardiotóxico por los agregados prefibrilares de cadenas ligeras, causando necrosis de los cardiomiocitos. ${ }^{6}$ Podrían verse afectados otros órganos vitales como riñones, hígado, sistema nervioso periférico y autonómico, al igual

Tabla 1: Tipos de amiloidosis con involucro cardiaco.

\begin{tabular}{|c|c|c|c|c|c|}
\hline Nomenclatura & $\begin{array}{l}\text { Proteína } \\
\text { precursora }\end{array}$ & $\begin{array}{l}\text { Rango } \\
\text { de edad }\end{array}$ & Sexo & $\begin{array}{l}\text { Cuadro } \\
\text { clínico }\end{array}$ & $\begin{array}{l}\text { Alteraciones } \\
\text { en laboratorio }\end{array}$ \\
\hline$A L$ & $\begin{array}{l}\text { Cadenas } \\
\text { ligeras }\end{array}$ & > 50 años & Cualquiera & $\begin{array}{l}\text { Involucro multiorgánico. Afectación } \\
\text { periorbital o macroglosia como } \\
\text { hallazgos patognomónicos } \\
\text { agregado a imágenes típicas en } \\
\text { RM o ecocardiograma. Hipotensión } \\
\text { severa con IECAS }\end{array}$ & $\begin{array}{l}\text { Elevación lambda o } \\
\text { kappa libre sérica con } \\
\text { radio anormal. Pico } \\
\text { monoclonal en suero } \\
\text { y/u orina. Supresión } \\
\text { de inmunoglobulinas. } \\
\text { Proteinuria }\end{array}$ \\
\hline ATTRwt & Transtiretina & > 65 años & $\begin{array}{l}\text { Predominancia } \\
\text { masculina }>15: 1\end{array}$ & $\begin{array}{l}\text { Historia de síndrome de túnel del } \\
\text { carpo sin otro órgano involucrado }\end{array}$ & $\begin{array}{c}\text { Sin anormalidades } \\
\text { específicas }\end{array}$ \\
\hline ATTRm & $\begin{array}{l}\text { Transtiretina } \\
\text { con } \\
\text { mutaciones }\end{array}$ & $\begin{array}{l}>40 \text { años } \\
\text { (dependiendo } \\
\text { mutación) en } \\
\text { afroamericanos } \\
60-65 \text { años }\end{array}$ & $\begin{array}{l}\text { Cualquiera, } \\
\text { predominancia } \\
\text { leve masculina }\end{array}$ & $\begin{array}{c}\text { Origen afroamericano/Caribe } \\
\text { (variante } \mathrm{V} 122 \mathrm{I} \text { ) }\end{array}$ & $\begin{array}{c}\text { Sin anormalidades } \\
\text { específicas. Estudios } \\
\text { genéticos muestran } \\
\text { mutación en molécula } \\
\text { TTR }\end{array}$ \\
\hline $\begin{array}{l}\text { AA } \\
\text { (secundaria) }\end{array}$ & $\begin{array}{l}\text { Amiloide A } \\
\text { sérico }\end{array}$ & $\begin{array}{l}\text { Entre } 20-30 \text { años } \\
\text { con enfermedad } \\
\text { inflamatoria } \\
\text { severa }\end{array}$ & Cualquiera & $\begin{array}{l}\text { Enfermedad crónica inflamatoria. } \\
\text { Hepato/esplenomegalia. No } \\
\text { involucro cardiaco, en casos raros } \\
\text { puede ser severo }\end{array}$ & $\begin{array}{c}\text { Proteinuria. Elevación } \\
\text { VSG/PCR }\end{array}$ \\
\hline
\end{tabular}

$\mathrm{AL}=$ amiloidosis de cadenas ligeras; ATTRwt = amiloidosis por transtiretina tipo silvestre; $\mathrm{ATTRm}=$ amiloidosis por transtiretina mutante; $\mathrm{AA}=$ amiloidosis adquirida; IECAS = inhibidores de enzima convertidora de angiotensina; RM = resonancia magnética; VSG = velocidad de sedimentación globular; $P C R$ = proteína $C$ reactiva.

Modificada de: Falk RH, Alexander KM, Liao R, Dorbala S. AL (light-chain) cardiac amyloidosis: a review of diagnosis and therapy. J Am Coll Cardiol. 2016; 68 (12): 1323-1341. 
que tejidos blandos. ${ }^{7}$ Este tipo de amiloidosis se caracteriza por ser de rápida progresión, por lo que es importante realizar un diagnóstico temprano. ${ }^{4}$

El cuadro clínico depende del depósito de amiloide multisistémico; dentro de la exploración física, la infiltración a tejidos blandos y pequeños vasos se podría presentar como macroglosia, púrpura periorbitaria, distrofia nasal e hipertrofia de glándulas submandibulares, si ocurre infiltración hepática o esplénica se evidencia a manera de organomegalia, la afectación renal es común y usualmente se presenta con proteinuria en rango nefrótico llevando a enfermedad renal progresiva. ${ }^{2}$ Es común la neuropatía periférica con apariencia de parestesias o disestesias de predominio "guante y calcetín", la neuropatía autonómica se revela como hipotensión ortostática, cambios en patrón de evacuaciones y disfunción eréctil. En etapas tempranas, la forma cardiaca es un reto diagnóstico, ya que la sintomatología de falla cardiaca derecha no es evidente hasta que la enfermedad se encuentra en estadios avanzados, se puede presentar con presión venosa yugular aumentada, tercer ruido, hepatomegalia y edema periférico; estas manifestaciones podrían estar ausentes en enfermos en los que se inició manejo con diurético, ${ }^{7}$ también pueden presentar cambios en la frecuencia de latidos, por lo que podrían llegar a presentar palpitaciones, síncope o muerte súbita, puede haber infiltración a válvulas cardiacas causando alteración en las tricúspide y mitral. ${ }^{2}$

Dentro de los estudios diagnósticos es posible identificar inmunoglobulina monoclonal o cadenas ligeras en suero y/u orina en un radio $3: 1^{5}$ en por lo menos $95 \%$ de los pacientes; sin embargo, en electroforesis sérica no son reportados, lo cual es problemático para el diagnóstico y monitoreo de respuesta al tratamiento. ${ }^{7}$

\section{AMILOIDOSIS POR TRANSTIRETINA}

La transtiretina es una proteína plasmática sintetizada y secretada en el hígado y plexo coroideo, la cual funciona a modo de transportador de tiroxina y proteína de unión a retinol. ${ }^{5}$ Es viable dividir esta forma de amiloidosis en dos tipos: hereditaria (mutante) y silvestre. En su presentación silvestre, antes referida como sistémica senil por su inicio en etapas tardías de la vida, muestra predominancia en hombres. Por lo general, el corazón es el único órgano afectado dentro de esta enfermedad, no obstante, se ha observado involucro de ligamentos y del espacio tenosinovial causando síndrome del túnel carpiano ${ }^{5}$ y otros musculoesqueléticos, por ejemplo estenosis del canal lumbar, el cual se ha visto puede preceder a la sintomatología cardiaca por 10 a 15 años. Se considera un padecimiento neurológico, sin embargo, por los registros mundiales se sugiere que el corazón es el órgano más afectado en por lo menos la mitad de los casos. ${ }^{11}$ Su prevalencia ha aumentado por la edad de la población actual, al igual que el avance en herramientas diagnósticas entre las que se encuentran la resonancia magnética cardiovascular y la cintigrafía Tc-DPD, pese a que la biopsia del corazón es necesaria en estos pacientes buscando obtener un diagnóstico definitivo. ${ }^{7}$

Se han encontrado pequeños depósitos amiloides de transtiretina estructuralmente inestable en autopsias de individuos de edad avanzada, este porcentaje va de un 14\%-25\%, dependiendo de la selección del paciente y del método de detección utilizado. ${ }^{12}$

En su presentación hereditaria, en el cromosoma 18 se localiza gen TTR, del cual existen más de 120 mutaciones, la mayoría codifica variantes patogénicas que aumentan el potencial amiloidogénico, las variantes responsables de la mayoría de los casos son estas: la Val30Met, que es la causa más común en el mundo de polineuropatía amiloide familiar, que se revela como neuropatía periférica y autonómica sin envolvimiento cardiaco; Thr60Ala es la que se presenta como una cardiomiopatía caracterizada por falla cardiaca y alteración en el sistema de conducción, alteración autonómica o neuropatía periférica o en combinación; Ser77Tyr y Val122lle son mutaciones que presentan un patrón autosómico dominante con penetrancia variable. La amiloidosis se presenta entre la tercera y cuarta década de la vida, clínicamente se podría manifestar como neuropatía periférica o autonómica y las anormalidades del corazón se pueden encontrar ausentes o limitadas a alteraciones en el sistema de conducción ${ }^{7}$ y valvulopatías, especialmente estenosis aórtica, la cual es observada en un $14 \%-16 \%$ en pacientes ancianos en los que se considera reemplazo de válvula aórtica transcatéter (TAVR, por sus siglas en inglés) y en $6 \%$ de los enfermos que se someterán a reemplazo de válvula. ${ }^{13}$

En el Instituto Nacional de Ciencias Médicas y Nutrición "Salvador Zubirán" se llevó a cabo un estudio del 2010 al 2017, en donde se seleccionaron enfermos sospechosos de amiloidosis tipo hereditaria, y se encontró que hay un foco endémico, sobre todo en los estados de Morelos y Guerrero, esta investigación evidenció mutaciones (Ser50Arg en un $74 \%$, Gly47Ala en 13\%, Ser52Pro en $11 \%$ y V122I/ $\mathrm{Y} 116 \mathrm{H}$ en $2 \%$ ) que fueron distintas de la más común de manera global que es la Val30Met; se hace referencia a la falta de más exploraciones a fin de determinar la prevalencia de la amiloidosis familiar en este país y así ofrecer consejería genética e iniciar tratamiento que permita la mejoría en la calidad y expectativa de vida. ${ }^{14}$

La presentación silvestre, previamente conocida como senil, es causada por acumulación de fibrillas proteicas de transtiretina mal plegadas, cuando los depósitos son mayores y principalmente en el corazón se sospecha este tipo de amiloidosis, no obstante, su mecanismo aún no es del todo claro. ${ }^{8}$ El diagnóstico de amiloidosis por transtiretina, 
en cualquiera de sus formas, es más complicado ya que la presentación clínica y la severidad del daño orgánico es gradual y variable. Se ha verificado que del $10 \%$ al $25 \%$ de los pacientes con falla cardiaca con fracción de eyección preservada presentan un acopio de transtiretina de tipo silvestre en ventrículo izquierdo, al momento de realizar autopsia. $^{15}$

Dentro de la presentación clínica, es plausible encontrar hipertrofia de ventrículo izquierdo con presión de llenado elevada y falla del corazón con fracción de eyección preservada, se acompañan de disnea, disminución de tolerancia al ejercicio, fatiga, edema de miembros pélvicos y/o abdominal, saciedad temprana y disfunción eréctil. ${ }^{15} \mathrm{El}$ dolor anginoso se puede presentar por infiltración microvascular en enfermos sin estenosis obstructiva coronaria. Se debe tener en cuenta que estos pacientes pueden presentar, desde años previos al diagnóstico, síndrome del túnel carpiano y estenosis del canal lumbar, al igual que depósitos en hígado y pulmón sin ser de relevancia clínica. ${ }^{8,15} \mathrm{Al}$ surgir en personas de edad avanzada, no es raro encontrar fibrilación auricular asociada con esta enfermedad, se sugiere que esta arritmia es más común en este tipo de amiloidosis que en otras etiologías de anomalía cardiaca. ${ }^{16}$

La baja tasa de diagnóstico se debe a la naturaleza del trastorno, ya que se presenta en población de edad avanzada, quienes frecuentemente cuentan con comorbilidades y se cree que el diagnóstico no afectará el pronóstico del paciente. ${ }^{15}$ Se realizó un estudio que incluyó 146 pacientes en el Centro de Amiloidosis de la Universidad de Boston entre junio de 1994 y enero de 2014, en donde se encontró que la fibrilación auricular ocurre en la mayoría de los afectados con amiloidosis por transtiretina tipo silvestre $(70 \%)$, presentando más irregularidades diastólicas, sin embargo, la fibrilación auricular no parece impactar la tasa de supervivencia en estos pacientes. ${ }^{16}$

La sospecha clínica es muy importante para el diagnóstico oportuno, algunos puntos clave son los siguientes: hipertrofia de ventrículo izquierdo sin historia previa de hipertensión arterial sistémica severa o mal controlada, hipotensión de reciente aparición, intolerancia a inhibidores de la enzima convertidora de angiotensina (IECA) o $\beta$ bloqueadores, arritmias atriales, alteración en la función nodal la cual requiera de marcapasos permanente y la presencia de síndrome del túnel carpiano bilateral y/o estenosis lumbar. ${ }^{15}$

Otro tipo de la enfermedad es la AA (amiloide A), previamente conocida como amiloidosis adquirida, secundaria o reactiva, la que es causada por la proteína amiloide A y se puede presentar en el contexto de un proceso inflamatorio crónico o infeccioso, como padecimiento inflamatorio intestinal, artritis reumatoide, osteomielitis, fiebre familiar mediterránea, tuberculosis y endocarditis subaguda. ${ }^{4}$
Existen tres tipos adicionales de proteínas relacionadas con la apolipoproteína (apolipoproteína A-I, apolipoproteína II y apolipoproteína IV), mismas que se han asociado con amiloidosis sistémica. Se divide en dos tipos, el hereditario que presenta afectación renal, hepática y cardiaca y la no hereditaria que se relaciona con tejido blando y vasculatura. $^{3}$

La forma amiloide $\mathrm{A} \beta 2 \mathrm{M}$ es resultado del mal plegamiento de la microglobulina $\beta 2$, la cual ocurre en aquejados por una disfunción renal de larga evolución en terapia dialítica, estos depósitos se acumulan alrededor de articulaciones y otros tejidos cuando no pueden ser eliminados por la presencia de falla renal. ${ }^{4}$ La variante $A \beta$ amiloide es la más común de amiloidosis localizada y es encontrada dentro del sistema neurológico en pacientes con enfermedad de Alzheimer. ${ }^{4}$

La forma AAI (amiloidosis atrial aislada) es una condición de gran importancia para la patofisiología de arritmias que se originan en la aurícula como fibrilación auricular, ésta es causada por acumulación de péptido natriurético atrial (PNA) en las aurículas. Parece ser el tipo más común del trastorno, que afecta el tejido cardiaco, ya que más del 90\% de los enfermos mayores de 90 años presentan depósitos de PNA, su predilección por mujeres se podría explicar por el hecho que el estradiol regula la expresión PNA en los cardiomiocitos atriales. ${ }^{17}$

\section{DIAGNÓSTICO}

La cardiopatía por amiloidosis debe ser sospechada en pacientes que muestren falla del corazón rápidamente progresiva en la que se agregan síntomas de neuropatía o alteración autonómica, síndrome del túnel del carpo, y que haya sido abordada por medio de estudios de imagen, por ejemplo ecocardiograma y resonancia magnética cardiaca, intolerancia al uso de medicaciones cardiovasculares previamente toleradas, esto debe ser completado con análisis bioquímicos dirigidos a dicha patología, como proteínas séricas monoclonales, proteínas urinarias Bence Jones y cadenas ligeras séricas. ${ }^{18,19}$

En España, un centro hospitalario describe las características clínicas de 180 pacientes atendidos en alrededor de 10 años de las que sobresale la mayor frecuencia de anomalías del corazón y mortalidad en amiloidosis cardiaca por cadenas ligeras (Tabla 2). ${ }^{20}$

En la presentación por transtiretina se recomienda la secuencia genética en todos los casos, ya que no se pueden distinguir las formas adquiridas de las hereditarias en el contexto clínico, puede no estar presente dentro de los antecedentes heredofamiliares al ser una enfermedad autosómica dominante y tener variabilidad en la penetrancia. ${ }^{11}$ 
Tabla 2: Características diferenciales de pacientes con amiloidosis cardiaca.

\begin{tabular}{|c|c|c|c|}
\hline & ATTR $(N=116)$ & $\mathrm{AL}(\mathrm{N}=64)$ & \\
\hline Características basales & $\mathrm{n}(\%)$ & $\mathrm{n}(\%)$ & $\mathrm{p}$ \\
\hline Edad en años* & $79 \pm 12$ & $64 \pm 11$ & $<0.001$ \\
\hline Tiempo hasta diagnóstico en años* & $2.8 \pm 4.3$ & $0.6 \pm 0.7$ & $<0.001$ \\
\hline Varones & $83(71)$ & $32(50)$ & 0.004 \\
\hline Insuficiencia cardiaca & $51(44)$ & $50(80)$ & $<0.001$ \\
\hline Fibrilación atrial & $24(20)$ & $5(8)$ & 0.0280 \\
\hline NYHA III-1V al diagnóstico & $28(24)$ & $31(51)$ & $<0.001$ \\
\hline Portador de marcapaso & $24(20)$ & $3(4)$ & 0.004 \\
\hline Síndrome del túnel del carpo & $48(41)$ & $9(14)$ & $<0.001$ \\
\hline Disautonomía & $71(61)$ & $24(37)$ & 0.002 \\
\hline Afectación renal & $59(50)$ & $57(89)$ & $<0.001$ \\
\hline Mortalidad total a los 3 años & $37(31)$ & $31(48)$ & 0.028 \\
\hline
\end{tabular}

\section{ECOCARDIOGRAMA}

El análisis inicial de elección de tipo no invasivo es el ecocardiograma, en el cual se observa engrosamiento del septum interventricular o la pared del ventrículo izquierdo $>12 \mathrm{~mm},{ }^{2}$ disminución en volumen ventricular izquierdo, engrosamiento valvular, dilatación auricular y signos de presión elevada con llenado diastólico restrictivo, la fracción de eyección del ventrículo izquierdo es preservada en estadios tempranos, ${ }^{9}$ empero, si se realiza solamente este estudio no es posible distinguir entre las patologías que causan engrosamiento de la pared ventricular, por lo tanto se recomienda un abordaje multimodal. ${ }^{7}$ Se ha sugerido que la pobre sensibilidad y especificidad de este tipo de pesquisas pueden contribuir a la falta de diagnóstico o retraso del mismo. ${ }^{21}$

\section{ELECTROCARDIOGRAMA}

Se observan voltajes disminuidos conforme progresa la enfermedad debido a la infiltración miocárdica que causa engrosamiento de la pared ventricular, también se asocia con desviación extrema del eje cardiaco, otros hallazgos son anormalidades en la repolarización, patrón pseudoinfarto anterior inferior o lateral, hemibloqueo anterior izquierdo, anormalidades de la onda T no específicas y alteraciones en el ritmo como fibrilación auricular. ${ }^{7}$ La incidencia de trombosis auricular es elevada, por lo que se ha propuesto el uso de anticoagulación profiláctica a largo plazo y su inicio temprano puede reducir morbimortalidad. ${ }^{22}$

\section{BIOPSIA}

La biopsia endomiocárdica se mantiene en el puesto de estándar de oro ${ }^{23}$ para diagnóstico y evaluación del involucro cardiaco (enfermos postrasplantados, sospechosos de miocarditis, cardiomiopatía, toxicidad o en condiciones sistémicas en la cual se presume padecimiento cardiaco como son las enfermedades de depósito, ${ }^{2}$ ) se utiliza la tinción Rojo Congo, que produce la birrefringencia verde patognomónica al ser observado a través de luz polarizada, se pueden utilizar tinciones de tioflavina y azul sulfatado, ${ }^{4,5}$ si se realiza tinción con hematoxilina y eosina se observa material amorfo bajo el microscopio, ${ }^{4}$ es importante mencionar que este examen no determina el tipo de amiloide, por lo que se realiza una espectrometría de masa, con una sensibilidad del 98\%, ya que la inmunohistoquímica puede afectar el resultado. ${ }^{24}$ La biopsia de médula ósea debe ser el procedimiento estándar cuando se sospecha amiloidosis de cadenas ligeras, por la posibilidad de presentar falsos positivos en la tinción si se realiza en laboratorios no experimentados con esta dolencia. ${ }^{4}$

Los sedimentos amiloides suelen ser identificados en biopsia de órganos alterados, sin embargo, hay una alternativa al realizar biopsias no invasivas, por ejemplo en el aspirado de grasa abdominal es posible identificar depósitos en un $60 \%$ $80 \%$ de pacientes con enfermedad por cadenas ligeras, pero menos en la afectación por transtiretina. ${ }^{7}$ La combinación del aspirado de grasa abdominal y biopsia de médula ósea son más sensibles en la variante por cadenas ligeras. ${ }^{22}$ 
Como se mencionó previamente, la biopsia endomiocárdica ha sido el estudio estándar para el diagnóstico de este trastorno, si bien como aspectos negativos tiene el ser un proceso invasivo, en el cual al realizar biopsia solamente de una parte del corazón podría ser ineficaz tratando de realizar el diagnóstico, ya que los sedimentos amiloideos pueden no ser extensos o encontrarse difusos. Los métodos por imagen pueden ofrecer una vista completa del corazón y pueden ser repetidos fácilmente para asesorar la respuesta al tratamiento. ${ }^{24}$

En general, en los pacientes con hallazgos clínicos de amiloidosis se deben realizar exploraciones multiimagen y así confirmar el diagnóstico y cuantificar el involucro cardiaco procurando la óptima selección terapéutica y mejorar el pronóstico del paciente. ${ }^{25}$

\section{RESONANCIA MAGNÉTICA CARDIACA}

Tiene ventajas únicas en identificar el envolvimiento cardiaco, ya que al administrar contraste con gadolinio da imágenes patognomónicas de la enfermedad, como realce tardío subendocárdico o transmural, ${ }^{5}$ este tipo de estudio se utiliza en los afectados por engrosamiento de la pared del ventrículo izquierdo y/o hipertrofia, para así diferenciar las patologías celulares e intersticiales, lo cual no es posible con ecocardiografía. Dentro de los hallazgos por RM en amiloidosis por transtiretina se logra encontrar que en el $79 \%$ de los casos se observa hipertrofia asimétrica del ventrículo izquierdo. ${ }^{18}$ La desventaja del uso del gadolinio es que puede causar fibrosis sistémica nefrogénica, por lo que se contraindica en pacientes con afección renal moderada o severa. ${ }^{4}$

\section{CINTIGRAFÍA ÓSEA CON TECNECIO 99}

Este método es sensible a fin de identificar amiloidosis por transtiretina antes de presentar anormalidades en ecocardiograma, ${ }^{4}$ sin embargo, no es totalmente específico para esta condición, que es susceptible de ser diagnosticada en ausencia de prueba histológica cuando hay falla de corazón asociada confirmada por ecocardiograma o RM consistente con amiloidosis y cintigrafía grado 2 o 3, al igual que ausencia de Ig monoclonal sérica y urinaria, ${ }^{7}$ esta combinación aumenta la especificidad de un $87 \%$ a $100 \%$ en pacientes con biopsia endomiocárdica. ${ }^{21}$ La cuantificación de tecnecio 99 podría ser utilizada intentando determinar la severidad del involucro cardiaco por transtiretina; no obstante, el ecocardiograma se mantiene como el método estándar, un resultado negativo puede excluir la variante cardiaca o sugerir que la proteína transtiretina no es la causa del trastorno. ${ }^{4}$

\section{PET (TOMOGRAFÍA POR EMISIÓN DE POSITRONES)}

Se han evaluado varios marcadores de unión amiloideos en el PET para apoyar el diagnóstico de esta afección en sus presentaciones por transtiretina y de cadenas ligeras, el compuesto F-florbetapir, F-florbetapen y F-flutemetamol y $\mathrm{B}$ de C-Pittsburgh ${ }^{18}$ han sido usados en la imagen cerebral en la enfermedad de Alzheimer, puede detectar estos tipos de amiloidosis al unirse con la estructura B de las fibrillas amiloideas, lo cual facilita la identificación de los depósitos independientemente del precursor. ${ }^{13,18}$

\section{ESTUDIOS GENÉTICOS}

En el caso de amiloidosis por transtiretina, se puede confirmar mediante exámenes genéticos en los cuales se revela la mutación específica, se recomienda en pacientes sin antecedentes heredofamiliares de la enfermedad y que presenten síntomas atípicos. ${ }^{2}$

\section{BIOMARCADORES}

Dentro de los biomarcadores, los de tipo cardiaco como BNP, NT-pro-BNP sérico y troponina T, pueden ser útiles en denotar la magnitud del involucro cardiaco, estratificación de pronóstico y guiar las estrategias de tratamiento en amiloidosis por cadenas ligeras. ${ }^{2,7}$ Dichos NT-pro-BNP sérico y troponina T integran la clasificación Mayo (grado 0: ambos negativos, grado 1: un parámetro elevado, grado 2: ambos elevados). ${ }^{13}$ Se ha observado que el marcador pro BNP tiene $90 \%$ de especificidad y $92 \%$ de sensibilidad a fin de predecir anormalidades ecocardiografías y ayuda a determinar el inicio del seguimiento ecocardiográfico. ${ }^{4}$ Se ha observado que los marcadores NT-pro-BNP y la tasa de filtración glomerular pueden ser utilizadas para estatificación de la forma causada por transtiretina. ${ }^{13}$

\section{ULTRASONIDO DE TEJIDO GRASO}

Se han evaluado nuevos métodos diagnósticos, entre los que se encuentra la ultrasonografía (USG) de tejido graso para realizar tamizaje en pacientes con amiloidosis por transtiretina; en una investigación realizada por Misumi y colaboradores en $2017,{ }^{17}$ se reportó una sensibilidad del $85 \%$ y especificidad del $97 \%$, el cual presenta varias limitantes, entre ellas el tamaño de muestra y estructura del estudio, sin embargo, abre la posibilidad de ser considerada una herramienta de tamizaje.

\section{TRATAMIENTO}

En general, la amiloidosis por cadenas ligeras tiene un pobre pronóstico, en contraste, la variedad por transtiretina (he- 
reditaria y silvestre) se asocia con manifestaciones clínicas medias y progresión lenta, llevando a un mejor pronóstico; se ha observado que la amiloidosis por transtiretina presenta mejor evolución. ${ }^{4}$

Es plausible dividir el tratamiento en tres tipos: de soporte, modificadores de la enfermedad e inmunoterapia. Dentro del tratamiento de soporte se recomienda educar al paciente sobre su trastorno, así como establecer restricción de líquidos y sal. ${ }^{1,7}$ Dentro del manejo farmacológico de la falla cardiaca se utilizan combinaciones de diferentes tipos de diuréticos, principalmente de asa, ${ }^{9}$ teóricamente, los fármacos IECAS/ARAll y los antagonistas de mineralocorticoides pueden disminuir el remodelamiento cardiaco al suprimir el sistema RAA (renina-angiotensina-aldosterona) de manera sistémica y local; sin embargo, estos medicamentos no son bien tolerados y pueden causar hipotensión sistémica por el bajo gasto cardiaco y disfunción neurohumoral, los bloqueadores de canales de calcio también se encuentran contraindicados por sus efectos inotrópicos negativos, ${ }^{4}$ así mismo el uso de betabloqueadores, ya que su uso es complicado porque exacerba el bajo gasto y las alteraciones en la conducción, ${ }^{19}$ la hipotensión ortostática puede ser tratada con midodrina y medias de compresión, se menciona la fludrocortisona para intentar aumentar la retención de líquidos.?

Además de la falla del corazón, se pueden presentar bradiarritmias y arritmias ventriculares causando la muerte en estos pacientes, por lo que se considera que la colocación profiláctica de un marcapasos o desfibrilador cardiaco pudieran ser beneficiosos, sin embargo, este abordaje debe ser personalizado a cada paciente, ya que se necesitan más estudios buscando elucidar los criterios apropiados para la colocación de este tipo de dispositivos en cada subtipo de enfermedad. ${ }^{19}$ En términos generales, el marcapasos puede considerarse una opción en enfermos sintomáticos con bloqueo avanzado, sin embargo, el uso del desfibrilador implantable no parece tener indicaciones hacia la prevención de muerte cardiaca súbita, ya que la principal causa de muerte es la disociación electromecánica, ${ }^{9}$ se ha propuesto un algoritmo por el Centro Amiloide de Stanford, en el cual se considera el desfibrilador implantable en pacientes que se encuentran en un estadio NYHA menor a IV y que su expectativa de vida sea mayor a un año, cuando haya antecedente de síncope exercional o taquicardia ventricular documentada en monitoreo con Holter ambulatorio. ${ }^{26}$

Otro tipo de arritmias presentes son las auriculares, por lo que se considera iniciar anticoagulación con antagonistas de vitamina $\mathrm{K}$ o anticoagulantes orales, sin embargo, al no encontrar suficiente evidencia sobre su seguridad y eficacia, se deben tomar en cuenta las mismas contraindicaciones y reducción de dosis al igual que en otras dolencias. ${ }^{26}$
En amiloidosis por cadenas ligeras, el tratamiento de elección es quimioterapia dirigida a la población de células clonales. La combinación de bortezomib, inhibidor de la proteasa, con dexametasona y un agente alquilante como ciclofosfamida, ${ }^{5}$ es el esquema más usado en la primera línea de tratamiento en cadenas ligeras y se asocia con una tasa de respuesta hematológica del $90 \%$, con el $60 \%$ alcanzando una réplica parcial o completa, el bortezomib se liga con retención de líquidos y síntomas de tipo congestivos. ${ }^{4}$ También se puede utilizar melfalán a altas dosis con trasplante autólogo de células madre, ya que se asocian a remisiones más duraderas, teniendo en cuenta que muestra como restricción el alto riesgo de mortalidad en personas ancianas y en aquellos con enfermedad cardiaca avanzada o envolvimiento del sistema nervioso autónomo. Ixazomib es un inhibidor de proteosoma que se encuentra en ensayo clínico fase tres en pacientes con modalidad de cadenas ligeras refractaria o que presentan recaída, el carfilzomib también se encuentra bajo estudio. ${ }^{27} \mathrm{El}$ monitoreo frecuente de cadenas ligeras permite observar la consecuencia hematológica y realizar modificaciones rápidamente en caso de ser inefectivo, es importante tener en mente que casi el $25 \%$ de los afectados muere en los primeros meses posterior al diagnóstico. ${ }^{7} \mathrm{La}$ lenalidomida previene la acumulación de cadenas ligeras; aunque no es cardiotóxico, se asocia con acumulación de pro-BNP y aumento en niveles de troponina. ${ }^{4}$

El anticuerpo monoclonal anti-CD38, daratumumab, es otra opción de tratamiento que ha demostrado tasas de respuesta hematológica completa, ${ }^{27}$ lo cual lo convierte en un manejo ideal en este tipo de padecimiento. En la Universidad de Stanford se realizó un sondeo retrospectivo en pacientes tratados con daratumumab por 12 meses, en quienes había involucro cardiaco y renal en un $72 \%$ y $68 \%$ respectivamente, la respuesta hematológica fue de $76 \%$, con una tasa de reacción completa del $36 \% .{ }^{28}$ La Clínica Mayo utilizó este manejo en pacientes con enfermedad refractaria o en recaída y se observó una respuesta hematológica del $80 \%$ en 30 de ellos. ${ }^{29,30}$

Los criterios de elegibilidad para trasplante de células madre incluyen: edad menor a 65 años, Karnofsky 80\% o mayor, función cardiaca adecuada (presión sistólica > $90 \mathrm{mmHg}$, fracción de eyección $>45 \%$, troponina $\mathrm{T}<$ $0.06 \mathrm{ng} / \mathrm{mL}$, NT-pro-BNP $<8,500 \mathrm{pg} / \mathrm{mL}$ o saturación de arteria pulmonar $>55 \%$ ) y cambios ortostáticos controlados o compensados en presión sanguínea. El trasplante autólogo de médula ósea se contraindica en enfermos con clase 3 y 4 de falla cardiaca en la escala NYHA, ya que los agentes quimioterapéuticos son cardiotóxicos o empeoran la sintomatología, así como estadio III en la clasificación de Mayo o afección renal crónica estadio IV. ${ }^{11}$

En amiloidosis por transtiretina en su forma hereditaria, el tratamiento de elección en personas jóvenes es el 
trasplante de hígado, procurando eliminar la fuente de la variante Val30Met; puede ser beneficioso para los otros tipos de mutaciones; sin embargo, la afectación del corazón presente al momento del trasplante hepático continúa progresando, en ciertos casos seleccionados se puede realizar un trasplante cardiaco pero la mayoría de los pacientes son de mayor edad para poder ser seleccionados.

En la actualidad, estudios in vitro sugieren estrategias en las cuales se produzca bloqueo en la producción de proteínas transtiretina en el hígado, interfiriendo con la disociación de proteínas al estabilizar el tetrámero TTR y degradando las fibrillas amiloides. ${ }^{5,27}$ Hay ensayos fase II y III con los siguientes esquemas: tafamidis (AINE derivado del benzoxazol), diflunisal (AINE), mismos que actúan en el papel de estabilizadores de esta proteína al inhibir la disociación a monómeros y así disminuir los agregados fibrilares y el tejido de deposición de proteína, finalmente, una combinación de doxiciclina con ácido ursodesoxicólico con la meta de disminuir de manera sinérgica la deposición de transtiretina y así disminuir la progresión de la enfermedad neuropática. ${ }^{27} \mathrm{Al}$ ser exclusivamente sintetizado en el hígado, se han desarrollado terapias inhibitorias de ARN y antioligonucleótido, ya que se ha visto que ellas pueden reducir la producción en más del $80 \% .^{7}$ El patisirán es una de las nuevas terapias que limita la producción de transtiretina interfiriendo en el ARN del hepatocito causando hendiduras antes de la traducción de proteínas. ${ }^{27}$ En el ensayo clínico APOLLO, se comparó patisirán contra placebo y se observó un beneficio sobre las manifestaciones de la neuropatía autonómica, neuropatía sensomotora así como mejoría en la calidad de vida. ${ }^{31}$

La inmunoterapia tiene el potencial de atacar los depósitos amiloideos directamente, como se ha observado con el anticuerpo quimérico Mu11-1F4, el cual se une a las fibrillas constituyentes de amiloidosis de cadenas ligeras, y que ha completado la fase I de ensayo clínico; otro anticuerpo monoclonal, NEOD001, que superó las fases I y II, ha evidenciado seguridad y tolerabilidad, sugiriendo mejora cardiaca, y actualmente se encuentra en fase III. Por último, el componente sérico amiloide $\mathrm{P}$ se une a todas las fibrillas amiloideas in vitro y las protege de degradación para después ser atacado por anticuerpos monoclonales anti SAP, al término de la fase I se observó reducción rápida de cúmulos amiloideos en el hígado, sin presentar efectos adversos importantes, actualmente se encuentra en fase II. ${ }^{7}$

En los aquejados por falla severa del corazón, secundaria a amiloidosis cardiaca aislada, se puede considerar la posibilidad de trasplante, aunque solamente se reserva a pacientes que sean considerados de alto riesgo para iniciar la quimioterapia intensiva requerida para el trasplante de células madres y que tengan esta presentación aislada ya que, en la presentación por cadenas ligeras, los enfermos tienen una carga significativa en otros órganos, por lo que no pueden ser elegidos. ${ }^{9}$ En un estudio realizado en el Hospital General de Massachusetts, Boston, se corroboró que el trasplante ortotrópico cardiaco seguido de trasplante autólogo de células madres mejoraba la sobrevivencia en el seguimiento a 4.6 años sin evidencia de enfermedad recurrente. ${ }^{32}$ En la variedad causada por transtiretina se necesitan trasplantes hepático y cardiaco, ya que el hígado es el sitio de producción de la proteína mutada, estos procedimientos tienen mejores resultados en dicha variedad de pacientes. ${ }^{4}$

La respuesta hematológica al tratamiento es susceptible de ser medida por la diferencia en la concentración entre inmunoglobulinas de cadena ligera libres involucradas y no involucradas en suero; la respuesta parcial se define como la disminución en la diferencia en un 50\%, buena consecuencia si las cadenas ligeras son $<40 \mathrm{mg} / \mathrm{L}$ y alcanza a ser completa si se normaliza la diferencia con inmunofijación negativa en suero y orina. La reacción cardiaca puede ser valorada por cambios en los valores BT-pro-BNP; si es > $600 \mathrm{ng} / \mathrm{L}$ y hay reducción $>30 \%$ se considera un corolario adecuado. Es posible ver estas dos alternativas tres meses posteriores al inicio del tratamiento y ellas pueden ser predictoras de supervivencia. ${ }^{22}$

En un estudio realizado en 2016 por Kristen A y colegas, ${ }^{23}$ se observó que las tasas de mortalidad fueron significativamente menores en amiloidosis por transtiretina comparado con cadenas ligeras, en los pacientes que mostraban carga amiloide $<20 \%$ o $>40 \%$; presentaban menos sobrevivencia que en aquellos con carga entre 20$40 \%$, la tasa de supervivencia depende significativamente de la quimioterapia. Si se reportaba $<20 \%$ de la carga amiloide independiente de la respuesta, no se evidenciaba diferencia en la sobrevivencia. No se encontró diferencia si la carga amiloide era del $20-40 \%$ o $>40 \%$.

\section{CONCLUSIONES}

La amiloidosis cardiaca requiere de gran sospecha clínica y de apoyo de análisis séricos y de imagen para poder realizar el diagnóstico. Con el propósito de realizar una valoración de la misma, los avances en las técnicas de imagen, al igual que las nuevas propuestas no invasivas juegan un papel importante en la esperanza de vida de estos enfermos, dejando la biopsia como la forma de hacer el diagnostico de certeza con las limitantes ya mencionadas este manuscrito. El futuro del tratamiento de ésta y otras enfermedades será basado en nuevas inmunoterapias, esperando encontrar nuevas opciones para así poder ofrecer una mayor sobrevida a estos pacientes. 


\section{REFERENCIAS}

1. Donnelly JP, Hanna M. Cardiac amyloidosis: An update on diagnosis and treatment. Cleve Clin J Med. 2017; 84 (12 Suppl 3): 12-26.

2. Flodrova P, Flodr P, Pika T, Vymetal J, Holub D, Dzubak P et al. Cardiac amyloidosis: from clinical suspicion to morphological diagnosis. Pathology. 2018; 50 (3): 261-268.

3. Di Giovanni B, Gustafson D, Adamson MB, Delgado DH. Hiding in plain sight: cardiac amyloidosis, an emerging epidemic. Can J Cardiol. 2020; 36 (3): 373-383.

4. Alkhawam H, Patel D, Nguyen J, Easaw SM, Al-Sadawi M, Syed U et al. Cardiac amyloidosis: pathogenesis, clinical context, diagnosis and management options. Acta Cardiol. 2017; 72 (4): 380-389.

5. Sperry BW, Tang WHW. Amyloid heart disease: genetics translated into disease-modifying therapy. Heart. 2017; 103 (11): 812-817.

6. Tuzovic M, Yang EH, Baas AS, Depasquale EC, Deng MC, Cruz D et al. Cardiac amyloidosis: diagnosis and treatment strategies. Curr Oncol Rep. 2017; 19 (7): 46.

7. Martinez-Naharro A, Hawkins PN, Fontana M. Cardiac amyloidosis. Clin Med (Lond). 2018; 18 (Suppl 2): s30-s35.

8. Yamamoto $\mathrm{H}$, Yokochi T. Transthyretin cardiac amyloidosis: an update on diagnosis and treatment. ESC Heart Fail. 2019; 6 (6): 1128-1139.

9. Bhogal S, Ladia V, Sitwala P, Cook E, Bajaj K, Ramu V et al. Cardiac amyloidosis: an updated review with emphasis on diagnosis and future directions. Curr Probl Cardiol. 2018; 43 (1): 10-34.

10. Hernández-Reyes J, Galo-Hooker E, Ruiz-Delgado GJ, Ruiz-Argüelles G). Systemic immunoglobulin light-chain amyloidosis (AL) in Mexico: a single institution, 30-year experience. Rev Invest Clin. 2012; 64 (6 Pt 2): 604-608.

11. Maurer MS, Elliott P, Comenzo R, Semigran M, Rapezzi C. Addressing common questions encountered in the diagnosis and management of cardiac amyloidosis. Circulation. 2017; 135 (14): 1357-1377.

12. González-López E, Gagliardi C, Dominguez F, Quarta CC, de HaroDel Moral FJ, Milandri A et al. Clinical characteristics of wild-type transthyretin cardiac amyloidosis: disproving myths. Eur Heart J. 2017; 38 (24): 1895-1904.

13. Fontana M, Corovic A, Scully P, Moon JC. Myocardial amyloidosis: the exemplar interstitial disease. JACC Cardiovasc Imaging. 2019; 12 (11 Pt 2): 2345-2356.

14. González-Duarte A, Cárdenas-Soto K, Bañuelos CE, Fueyo O, Dominguez C, Torres B, et al. Amyloidosis due to TTR mutations in Mexico with 4 distincts genotypes in the index cases. Orphanet J Rare Dis. 2018; 13 (1): 107.

15. Halatchev IG, Zheng J, Ou J. Wild-type transthyretin cardiac amyloidosis (ATTRwt-CA), previously known as senile cardiac amyloidosis: clinical presentation, diagnosis, management and emerging therapies. J Thorac Dis. 2018; 10 (3): 2034-2045.

16. Mints YY, Doros G, Berk JL, Connors LH, Ruberg FL. Features of atrial fibrillation in wild-type transthyretin cardiac amyloidosis: a systematic review and clinical experience. ESC Heart Fail. 2018; 5 (5): 772-779.

17. Kyriakou P, Mouselimis D, Tsarouchas A, Rigopoulos A, Bakogiannis C, Noutsias $M$ et al. Diagnosis of cardiac amyloidosis: a systematic review on the role of imaging and biomarkers. BMC Cardiovasc Disord. 2018; 18 (1): 221.
18. Martinez-Naharro A, Baksi AJ, Hawkins PN, Fontana M. Diagnostic imaging of cardiac amyloidosis. Nat Rev Cardiol. 2020; 17 (7): 413426. Available from: http://dx.doi.org/10.1038/s41569-020-0334-7

19. Izumiya Y, Takashio S, Oda S, Yamashita Y, Tsujita K. Recent advances in diagnosis and treatment of cardiac amyloidosis. J Cardiol. 2018; 71 (2): 135-143.

20. López-Sainz A, Hernandez-Hernandez A, Gonzalez-Lopez E, Domínguez F, Restrepo-Cordoba MA, Cobo-Marcos M et al. Clinical profile and outcome of cardiac amyloidosis in a Spanish referral center. Rev Esp Cardiol (Engl Ed). 2021; 74 (2): 149-158.

21. Brownrigg J, Lorenzini M, Lumley M, Elliott P. Diagnostic performance of imaging investigations in detecting and differentiating cardiac amyloidosis: a systematic review and meta-analysis. ESC Heart Fail. 2019; 6 (5): 1041-1051.

22. Gertz MA, Dispenzieri A, Sher T. Pathophysiology and treatment of cardiac amyloidosis. Nat Rev Cardiol [Internet]. 2015; 12 (2): 91-102. Available from: http://dx.doi.org/10.1038/nrcardio.2014.165

23. Kristen AV, Brokbals E, Aus dem Siepen F, Bauer R, Hein S, Aurich M et al. Cardiac amyloid load: a prognostic and predictive biomarker in patients with light-chain amyloidosis. J Am Coll Cardiol. 2016; 68 (1): $13-24$

24. Dorbala S, Cuddy S, Falk RH. How to image cardiac amyloidosis: a practical approach. JACC Cardiovasc Imaging. 2020; 13 (6): 1368-1383.

25. Alexanderson-Rosas E, Escudero-Salamanca M, Garcia-Diaz JA, Alvarez-Santana R, Cano-Zarate R, Mamani-Tito J et al. Light-chain cardiac amyloidosis: A multimodality approach. J Nucl Cardiol [Internet]. 2020; 27 (6): 2432-2435. Available from: https://doi. org/10.1007/s12350-019-02017-y

26. Aimo A, Rapezzi C, Vergaro G, Giannoni A, Spini V, Passino C et al. Management of complications of cardiac amyloidosis: 10 questions and answers. Eur J Prev Cardiol. 2020: 2047487320920756.

27. Wolfson AM, Shah KS, Patel JK. Amyloid and the Heart. Curr Cardiol Rep. 2019; 21 (12): 164.

28. Kaufman GP, Schrier SL, Lafayette RA, Arai S, Witteles RM, Liedtke M. Daratumumab yields rapid and deep hematologic responses in patients with heavily pretreated AL amyloidosis. Blood. 2017; 130 (7): 900-902.

29. Abeykoon JP, Zanwar S, Dispenzieri A, Gertz MA, Leung N, Kourelis T et al. Daratumumab-based therapy in patients with heavily-pretreated AL amyloidosis. Leukemia [Internet]. 2019; 33 (2): 531-536. Available from: http://dx.doi.org/10.1038/s41375-018-0262-2

30. Gertz MA. Immunoglobulin light chain amyloidosis: 2018 Update on diagnosis, prognosis, and treatment. Am J Hematol. 2018; 93 (9): 1169-1180.

31. González-Duarte A, Berk JL, Quan D, Mauermann ML, Schmidt $\mathrm{HH}$, Polydefkis $\mathrm{M}$ et al. Analysis of autonomic outcomes in APOLLO, a phase III trial of the RNAi therapeutic patisiran in patients with hereditary transthyretin-mediated amyloidosis. J Neurol [Internet]. 2020; 267 (3): 703-712. Available from: https://doi.org/10.1007/ s00415-019-09602-8

32. Dey BR, Chung SS, Spitzer TR, Zheng H, Macgillivray TE, Seldin DC et al. Cardiac transplantation followed by dose-intensive melphalan and autologous stem-cell transplantation for light chain amyloidosis and heart failure. Transplantation. 2010; 90 (8): 905-911. 